Conclusion According to our results, CA in non-complicated cases can be safely performed outside of hospital setting by board-certified interventional neuroradiologists with high success rates and minimal complications that are comparable to MRA and CTA. Our result show that not only CA is safe in an office setting, but potentially safer than MRA and CTA when considering higher quality imaging, evaluation of collateral flow and diagnosis of many additional vascular diseases. Proper case selection for this setting plays an important role in achieving optimal results and minimizing complications.

Disclosures S. Razavi: None. E. Masangkay: None. N. Chelikam: None. U. Kelly-Tolley: None. L. Pierce: None. R. Malek: None. A. Padiar: None.

\section{E-159 FEASIBILITY AND SAFETY OF TRANSRADIAL ACCESS FOR PEDIATRIC NEUROINTERVENTIONS}

${ }^{1} V$ Srinivasan*, C Hadley, 770301, 'M Prablek, ' $M$ Lopresti, ${ }^{2} S$ Chen, ${ }^{2} E$ Peterson, ${ }^{3} \mathrm{~A}$ Sweid, ${ }^{3} \mathrm{P}$ Jabbour, ${ }^{4} \mathrm{C}$ Young, ${ }^{4} \mathrm{M}$ Levitt, ${ }^{5} \mathrm{~J}$ Osbun, ${ }^{1} \mathrm{~J}$ Burkhardt, ${ }^{1} \mathrm{~J}$ Johnson, ${ }^{1} \mathrm{P}$ Kan. ${ }^{1}$ Neurosurgery, Baylor College of Medicine, Houston, TX; ${ }^{2}$ Neurosurgery, University of Miami, Miami, FL; ${ }^{3}$ Neurosurgery, Thomas Jefferson University, Philadelphia, PA; ${ }^{4}$ Neurosurgery, University of Washington, Seattle, WA; ${ }^{5}$ Neurosurgery, Washington University School of Medicine, St. Louis, MO

\subsection{6/neurintsurg-2020-SNIS.191}

Background Diagnostic cerebral angiograms are increasingly being performed by transradial access (TRA) in adults, following in line with data from the coronary literature supporting fewer access-site complications. Despite this ongoing trend on TRA in neuroangiography, there has been no discussion of its use in the pediatric population. In fact, pediatric TRA for other endovascular intervention has scarcely been described even for coronary or other applications. This is the first dedicated study of transradial access for neuroangiography in pediatric patients.

Methods A multi-institutional series of consecutively performed pediatric transradial angiograms and interventions was collected. This included demographic, procedural, outcomes, and safety data. Data was prospectively recorded and retrospectively analyzed.

Results A total of 37 diagnostic angiograms and 24 interventions were performed in 47 pediatric patients, by 5 neurointerventionalists. Proximal and distal angiography were performed successfully for both diagnostic and interventional application (19 distal angiograms, 2 distal interventions). Clinically significant vasospasm occurred in 7 patients $(11.5 \%)$. Re-access was successfully performed in 7 patients a total of 11 times. Conversion to femoral access occurred in 6 cases $(9.8 \%)$. There were no access-related complications otherwise.

Conclusions Transradial access in pediatric patients is safe and feasible. It can be performed successfully in many cases but carries some unique challenges compared to the adult population. Despite the challenge of higher rates of vasospasm and conversion to transfemoral access, it is worth exploring further, given the potential benefits.

Disclosures V. Srinivasan: None. C. Hadley: None. M. Prablek: None. M. Lopresti: None. S. Chen: None. E. Peterson: None. A. Sweid: None. P. Jabbour: None. C. Young: None. M. Levitt: None. J. Osbun: None. J. Burkhardt: None. J. Johnson: None. P. Kan: None.

\section{E-160 BLOOD CLOT IDENTIFICATION AND COMPOSITION ASSESSMENT BY FAST SPIN-ECHO (FSE) T2WI AND T2* MAPPING}

Y Ding*, M Abbassi, J Felmlee, D Dai, R Kadirvel, D Kallmes, W Brinjikji. Radiology, Mayo Clinic, Rochester, MN

\subsection{6/neurintsurg-2020-SNIS. 192}

Purpose There are growing data to suggest that clot composition can impact revascularization outcomes and strategies in large vessel occlusion patients. In many centers, MRI is the primary modality of acute stroke imaging so identifying clot composition on MRI imaging may be important. We performed an in vitro study to determine the MR signaling characteristics of stroke clots of various compositions.

Methods Fifteen thrombus analogs of five compositions $(n=3$ for each composition) [Group A, fibrin-rich (95\% plasma:5\% RBCs); Group B, fibrin-rich (75\% plasma:25\% RBCs); Group C, intermediate (50\% plasma:50\% RBCs); Group D, RBC-rich (25\% plasma:75\% RBCs,) and Group E, RBC-rich (5\% plasma:95\% RBCs)] were scanned with fast spin-echo (FSE) T2WI (TR/TE 2500/101 milliseconds (ms)) and quantitative T2* mapping sequence. Signals from FSE T2WI were collected, and thrombus T2* relaxation time (TT2*RT) was measured in all the groups. Correlation between the thrombus-T2* relaxation time and red blood cell content was analyzed.

Results Signal intensity changed gradually from high (bright) to low (dark) from Group A to E gradually from all the 3 clots in each group, which indicated the signal intensity was decreased as the composition of RBC increased. The average TT2*RT decreased from $60 \mathrm{~ms}$ (green) to $25 \mathrm{~ms}$ (red) from fibrin-rich clot to RBC-rich clot (Group A to E), which
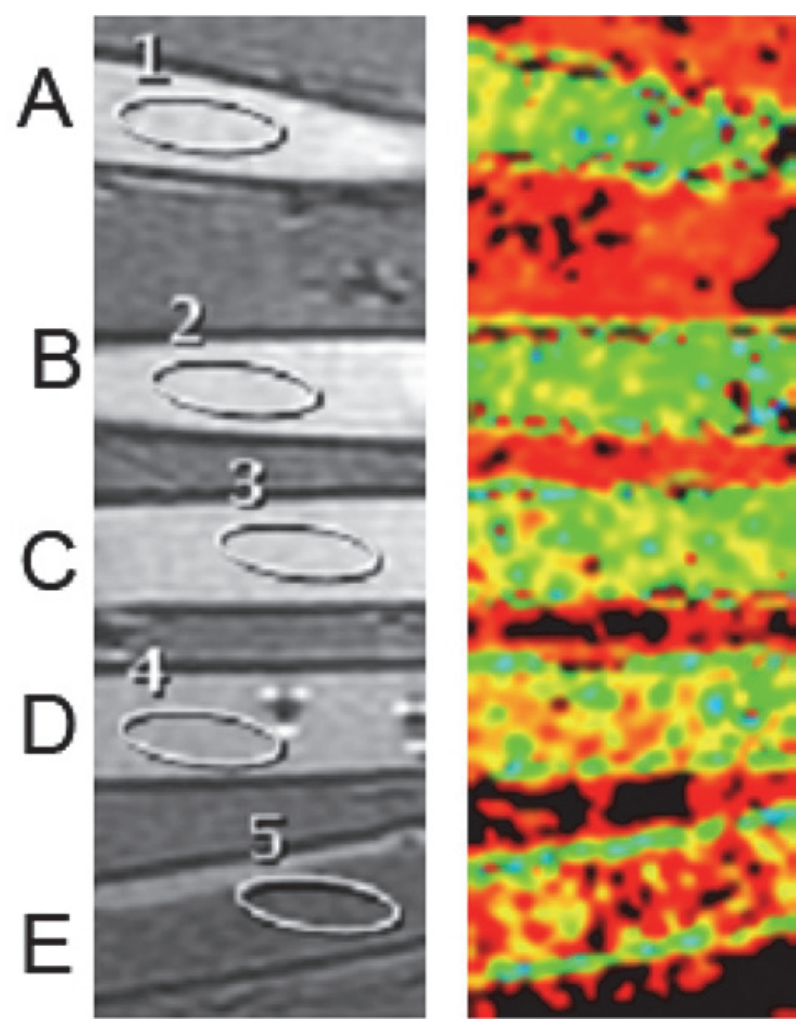

Abstract E-160 Figure 1 
reflected inverse correlation between thrombus-T2* relaxation time and red blood cell content (figures A to E).

Conclusion FSE T2 WI and quantitative T2* mapping MR can help in characterization of emboli in large vessel occlusion patients.

Disclosures Y. Ding: None. M. Abbassi: None. J. Felmlee: None. D. Dai: None. R. Kadirvel: None. D. Kallmes: None. W. Brinjikji: None.

\section{E-161 A NOVEL ANGIOGRAPHIC METHOD TO MEASURE ARTERIAL BLOOD FLOW RATES USING CONTRAST REFLUX}

${ }^{1} S$ Marfoglio, 'B Kovarovic, ${ }^{2} \mathrm{D}$ Fiorella, ${ }^{2} \mathrm{C}$ Sadasivan*. ${ }^{1}$ Biomedical Engineering, Stony Brook University, Stony Brook, NY; ${ }^{2}$ Neurological Surgery, Stony Brook University, Stony Brook, NY

\subsection{6/neurintsurg-2020-SNIS.193}

Introduction Several methods of extracting arterial blood flow rates from angiography have been attempted over the past decades. ${ }^{1} 2$ However, catheter-based contrast injections in arteries can cause substantial disturbances to the baseline blood flow, ${ }^{3}$ which has limited the utilization of these methods in the clinical setting. Contrast reflux, which is the movement of contrast proximal to the catheter tip is frequently observed during antegrade angiography. The goal of this study was to evaluate the relationship between baseline hemodynamics and contrast reflux.
Methods A silicone replica of a complete circle of Willis was connected to a pulsatile flow pump (Vascular Simulations, Stony Brook, NY). Contrast injections $(n=144)$ were performed in both the right carotid and right vertebral arteries under varying blood flow and injection conditions with highspeed (15 FPS) image acquisition. Reflux length was measured as the distance from the tip of the catheter to the most proximal point of contrast reflux. The reflux length was normalized by the vessel diameter at the catheter tip location and plotted against the ratio of the Reynolds number (a common fluid dynamics parameter) of blood flow to Reynolds number of contrast injection (figure 1). An appropriate equation was chosen to fit the data and the estimated mean blood flow from curve-fitting was compared to the true, measured blood flow. Mean blood flow was also estimated using the conventional Transit-Time method $^{1}$ and the flow estimation errors between the new and conventional methods were statistically compared.

Results The relationship between the Reynolds number ratio and reflux length could be described by a sigmoidal equation (figure $1, \mathrm{R}^{2}=0.64$ ). The Reflux method showed a lower error $(22.8 \pm 20.4 \%)$ as compared to the conventional Transit-Time method (31.7 $\pm 39.7 \%)$, but without statistical significance $(p=0.27)$.

Conclusion The novel Reflux method presented in this study may have better accuracy and precision than conventional transit-time methods for estimating blood flow. Contrast reflux at the catheter tip is essentially an output of hemodynamic disturbances caused by catheter injections with baseline flow
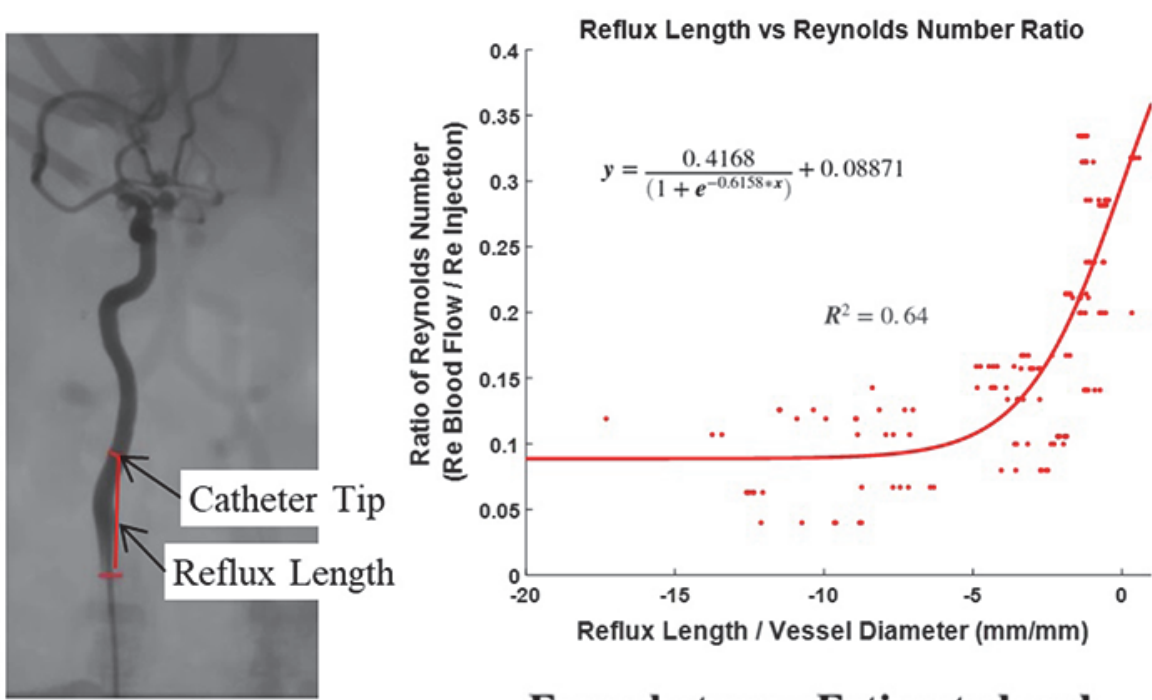

Error between Estimated and Measured Flow

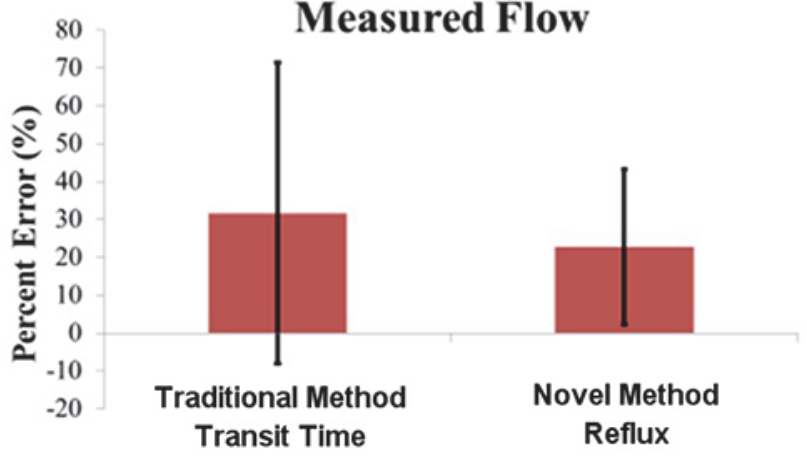

